\title{
APLICATIVO INFORMATIZADO COM O NURSING ACTIVITIES SCORE: INSTRUMENTO PARA GERENCIAMENTO DA ASSISTÊNCIA EM UNIDADE DE TERAPIA INTENSIVA
}

\author{
Meire Cristina Novelli e Castro ${ }^{1}$, Magda Cristina Queiroz Dell'Acqua ${ }^{2}$, José Eduardo Corrente ${ }^{3}$, Denise de \\ Cássia Moreira Zornoff ${ }^{4}$, Lucas Frederico Arantes ${ }^{5}$
}

\footnotetext{
${ }^{1}$ Mestre em Enfermagem. Enfermeira do Hospital de Clínicas da Faculdade de Medicina de Botucatu (FMB) da Universidade Estadual de São Paulo (UNESP). São Paulo, Brasil. E-mail: enfermeire.uti@fmb.unesp.br

${ }^{2}$ Doutora em Enfermagem. Professor Doutor no Departamento de Enfermagem da FMB/UNESP. São Paulo, Brasil. E-mail: mqueiroz@fmb.unesp.br

${ }^{3}$ Livre-docente. Professor Adjunto no Departamento de Bioestatística do Instituto de Biociências da UNESP. São Paulo, Brasil. E-mail: jecorren@ibb.unesp.br

${ }^{4}$ Médica Infectologista. Coordenadora do Núcleo de Educação à Distância e Tecnologias da Informação em Saúde da UNESP. São Paulo, Brasil. E-mail: dcmzornoff@fmb.unesp.br

${ }^{5}$ Aluno do Programa de Aprimoramento Profissional em Tecnologias da Informação Aplicada à Saúde, da UNESP. São Paulo, Brasil. E-mail: lfarantes@fmb.unesp.br
}

RESUMO: A gravidade do paciente ou o número de intervenções nem sempre serão proporcionais à carga de trabalho de enfermagem. Este estudo descritivo teve como objetivo demonstrar a trajetória da construção de um aplicativo (software) com o conteúdo do Nursing Activities Score e suas características operacionais. Foi realizado um teste piloto com 12 pacientes seguindo-se a coleta de dados por 90 dias consecutivos em 123 pacientes. Houve compatibilidade na transmissão de dados do Personal Digital Assistent para o computador de mesa, via wireless. A construção do aplicativo resultou em um sistema com coleta e administração de dados e permitiu realizar a interface gráfica. A utilização do aplicativo possibilita o uso de um sistema tecnológico para aplicação diária, com alimentação de um banco de dados sobre as características dos cuidados requeridos. Conhecendo a evolução destas variáveis durante a internação, o enfermeiro poderá planejar, intervir e avaliar a qualidade do cuidado.

DESCRITORES: Enfermagem. Unidade de terapia intensiva. Informática. Carga de trabalho. Nursing activities score.

\section{COMPUTER APPLICATION WITH THE NURSING ACTIVITIES SCORE: AN INTENSIVE CARE MANAGEMENT INSTRUMENT}

\begin{abstract}
Patients severity or the number of interventions will not always be proportional to the nursing staff's work load. This descriptive study aimed at demonstrating the trajectory for designing a computer application (software) with Nursing Activities Score content and its operational characteristics. A pilot test was conducted with 12 patients, which was followed by data collection from 123 patients for 90 consecutive days. Compatibility was observed in wireless data transmission from the Personal Digital Assistent to the desk computer. This application's construction resulted in a data collection and administration system, as well as enabled graphic interface. The use of the software allows for the utilization of a technological system with daily applications, with the support of a database concerning the characteristics of required care. With better understanding of the development of such variables during hospitalization, nurses will be able to plan, intervene, and evaluate care quality.
\end{abstract}

DESCRIPTORS: Nursing. Intensive care unit. Informatics. Workload. Nursing activities score.

\section{APLICATIVO INFORMATIZADO CON EL NURSING ACTIVITIES SCORE: INSTRUMENTO PARA GESTIÓN DE LA ATENCIÓN EN LA UNIDAD DE CUIDADOS INTENSIVOS}

RESUMEN: La gravedad de la enfermedad del paciente o el número de intervenciones no son siempre proporcionales a la carga de trabajo de la enfermería. Este estudio descriptivo tuvo como objetivo demostrar la trayectoria de la construcción de un aplicativo (software) con el contenido del Nursing Activities Score y sus características operacionales. Se realizó un test piloto con 12 pacientes y se recolectaron los datos por 90 días consecutivos en 123 pacientes. Hubo compatibilidad en la transmisión de datos del Personal Digital Assistent para la computadora de mesa inalámbrica. La construcción del aplicativo resultó en un sistema con recolección y administración de datos y permitió realizar la interfaz gráfica. La utilización del aplicativo posibilita el uso de un sistema tecnológico para aplicación diaria, con alimentación de un banco de datos sobre las características de los cuidados requeridos. Conociendo la evolución de estas variables durante la hospitalización, el enfermero podrá planificar, intervenir y evaluar la calidad del cuidado.

DESCRIPTORES: Enfermería. Unidad de terapia intensiva. Informática. Carga de trabajo. Nursing activities score. 


\section{INTRODUÇÃO}

Na perspectiva de responder algumas perguntas sobre a indicação de tratamento intensivo e nortear condutas, foram desenvolvidos e amplamente estudados índices específicos denominados como índices de gravidade. ${ }^{1}$ Com isto, permite-se muitas análises de diferentes alcances, podendo obter repercussões para a unidade, singularmente, mas também para outras unidades que realizem a aplicação destes escores.

Dentre os índices de gravidade os mais utilizados internacionalmente são o APACHE (Acute Physiologic and Chronic Health Evaluation) e o SAPS (Simplified Acute Physiologic Score) sendo, também, os mais acessados nas Unidades de Terapia Intensiva (UTIs). ${ }^{2-5}$ Entretanto, os índices de gravidade não são capazes de avaliar a carga de trabalho da equipe de enfermagem, pois são direcionados às condições clínicas dos pacientes e à terapêutica médica. Por isso, a gravidade do paciente ou o número de intervenções nem sempre serão proporcionais à carga de trabalho de enfermagem. ${ }^{6}$

$\mathrm{Na}$ aplicação de escores para medição da carga de trabalho de enfermagem foram constatados benefícios, nos quais, além da otimização dos recursos humanos da enfermagem, também podem ser observados ganhos na qualidade do atendimento aos pacientes, observada indiretamente pela diminuição de complicações, diminuição da morbidade, menor tempo de internação na UTI e na internação hospitalar, tendo impacto diretamente nos custos hospitalares. ${ }^{7}$

Em 2003, foi realizado um amplo estudo para ajustamento do TISS-28, escore mais utilizado para avaliar gravidade bem como carga de trabalho de enfermagem. O novo instrumento sofreu modificações, a partir do TISS-28, mas manteve sete grandes categorias: atividades básicas, suporte ventilatório, suporte cardiovascular, suporte renal, suporte neurológico, suporte metabólico e intervenções específicas. O escore resultante foi denominado Nursing Activities Score (NAS) e representa a porcentagem de tempo gasto por enfermeiro por turno, na assistência direta ao paciente. $\mathrm{O}$ instrumento abrange mais atividades desenvolvidas pela enfermagem e mostrou-se vantajoso por quantificar e graduar a complexidade do cuidado abrangendo $80,8 \%$ do tempo do profissional de enfermagem no cuidado do paciente no decorrer das 24 horas, ou seja, quase o dobro do TISS-28 (43,3\%). Seu objetivo é medir a carga real de trabalho de enfermagem na UTI, abrangendo o maior conjunto de atividades desenvolvidas pela equipe de enfermagem. $\mathrm{O}$ escore varia de 0 a 100 por cento ou mais, indica a porcentagem de tempo gasto pelo enfermeiro, por turno, na assistência de enfermagem direta. ${ }^{8}$

$\mathrm{O}$ índice originalmente descrito na língua inglesa foi traduzido e validado em 2002 e mostrou concordância com outros índices; assim o instrumento permite confiabilidade e validade para mensurar a carga de trabalho de enfermagem em UTI. ${ }^{9}$ O NAS proporciona condições para justificar e dimensionar a equipe de enfermagem de acordo com a carga de trabalho, podendo ser um instrumento utilizado para sistematizar e gerenciar o cuidado com qualidade. ${ }^{9}$ Considerando que cada ponto do NAS corresponde a 14,4 minutos, teremos a relação das horas necessárias para a assistência de enfermagem. ${ }^{10}$

A partir dos trabalhos realizados até o momento, a aplicação do NAS trouxe importantes possibilidades para a avaliação gerencial e assistencial das UTIs. O instrumento foi utilizado para levantamento das necessidades assistenciais em UTIs diferentes e para associação com a carga de trabalho. ${ }^{10-12}$ Recentemente foi empregado para estabelecer associação com indicadores de UTI como taxas de alta, óbito e readmissões. ${ }^{13}$ Também foi aplicado em neonatologia e obteve resultados favoráveis sobre sua utilização nesta área, fornecendo informações necessárias para o processo de trabalho relacionado a estes pacientes e outro estudo prospectivo registra com avaliação favorável. ${ }^{14-15}$

Em idosos sua aplicação não revelou diferença na demanda de trabalho de enfermagem para as diferentes faixas etárias e os fatores associados à alta carga de trabalho. ${ }^{16}$

A aplicação de instrumentos de mensuração da carga de trabalho de enfermagem em UTI em tempo real, imediatamente após a avaliação do paciente à beira do leito, tem sido um objetivo proposto para adequar a distribuição de pessoal de acordo com as demandas assistenciais da unidade. ${ }^{15}$ Acredita-se que a aplicação do NAS em um sistema informatizado auxilie na coleta de dados, processamento, arquivamento e monitorização dos resultados da assistência.

O exercício profissional do enfermeiro pauta-se na relação interpessoal enfermeiro-paciente-família-equipe. A utilização da tecnologia variavelmente parece tangenciar esta relação, trazendo algumas dúvidas, inseguranças e receios dos profissionais em se apropriar da tecnologia, como se fosse interferir negativamente na qualidade da assistência. Há evidências, que a incorporação tecnológica em nada fragmenta o cuidado, uma vez que a utilização destes recursos pode aperfeiçoar o 
registro e a transmissão da informação, adequandoos de forma mais clara, melhorando a comunicação e auxiliando na tomada de decisões. ${ }^{17}$

Para contribuir com a qualidade da assistência à saúde, um sistema de informações deve ser confiável, ter registro e transmissão em tempo hábil. O enfermeiro que intervêm no processo de trabalho por meio do computador, colabora com a prática de enfermagem e proporciona mudanças na gestão dessa assistência. ${ }^{18-19}$

O volume de informações sobre o paciente está cada vez maior e mais detalhado e pode ser observado pelo arsenal de exames que é possível realizar em uma internação. Portanto, o registro manual torna-se uma escolha insuficiente para contemplar todos os aspectos assistenciais. ${ }^{20}$

Com isto, por todos os trabalhos que utilizaram e evidenciaram a aplicação do NAS em diversas UTIs, acredita-se que há possibilidade da utilização de um aplicativo no gerenciamento da enfermagem, para aperfeiçoar a coleta de dados. A incorporação desta tecnologia no planejamento da assistência poderá redirecionar os cuidados, com ações de enfermagem específicas para pacientes atendidos em unidades de terapia intensiva.

Frente a essas reflexões é possível considerar que o referencial teórico sobre a mensuração da carga de trabalho, pelo do NAS $^{8}$ e a utilização da informática, podem resultar na construção de um instrumento eletrônico para coleta de dados e análise das necessidades assistenciais dos pacientes cuidados em UTI.

O objetivo deste estudo foi descrever a trajetória para a construção de um aplicativo (software) com o conteúdo do NAS $^{8}$ e suas características operacionais.

\section{MATERIAL E MÉTODO}

Tratou-se de um estudo descritivo, realizado em UTI geral do tipo III, conforme os critérios de classificação do Ministério da Saúde. ${ }^{21}$ Esta UTI está inserida no contexto de um Hospital Escola e tem como missão o ensino, a pesquisa e a assistência. A instituição representada pelo superintendente do Hospital das Clínicas - UNESP e os responsáveis pela UTI conheceram e aprovaram o projeto, que foi encaminhado para o Comitê de Ética e Pesquisa da Faculdade de Medicina de Botucatu e obteve parecer favorável OF $\mathrm{N}^{\circ}$ $600 / 2006$. Manteve-se o anonimato dos pacientes que originaram os dados e dos enfermeiros que utilizaram o instrumento informatizado.
O planejamento do sistema teve início com reuniões periódicas com a equipe técnica do Núcleo de Educação à Distância e Tecnologias da Informação em Saúde da Faculdade de Medicina de Botucatu UNESP, para definição do tema e funcionalidades desejadas. O conteúdo foi baseado no referencial teórico $\mathrm{NAS}^{8}$, o qual deveria ser adaptado para apresentação como formulário eletrônico de acesso restrito. $\mathrm{O}$ banco de dados seria instalado em estação de trabalho da unidade de terapia intensiva referida no estudo e alimentado em equipamento móvel do tipo PDA (Personal Digital Assistent).

A metodologia empregada no desenvolvimento do sistema foi a prototipação, um paradigma da engenharia de software baseado na criação de um modelo rápido, que permite identificar algumas etapas de interação entre o usuário e o aplicativo, seguido do refinamento do projeto. ${ }^{22}$

Seu desenvolvimento utilizou programas para edição de textos e imagens (Microsoft Word, Macromedia Fireworks, Corel Draw e Core Photo-Paint), geradores de gráficos (Microsoft Excel) e do código fonte do sistema (Macromedia Dreamweaver).

As páginas foram desenvolvidas estruturalmente em HTML (Hyper Text Markup Language) e ASP (Active Server Page) e as interatividades em Java Script. O banco de dados foi desenvolvido e gerenciado em MySQL (My Strutured Query Language).

A escolha da linguagem ASP deu-se devido a existência de bibliotecas que facilitam a programação do sistema para a manipulação de arquivos como planilhas e gráficos do Microsoft Excel, a disponibilidade do uso da linguagem, nativa do Microsoft Windows XP Professional e por não exigir a instalação de outros programas. A compatibilidade com o servidor (IIS) foi também considerada. O banco de dados MySQL foi selecionado por utilizar a linguagem SQL, a mais difundida para sistemas web, o que facilita o suporte. Além do exposto, o sistema é gratuito e permite um servidor muito estável. ${ }^{23}$

Para facilitar a mobilidade e a segurança na transmissão das informações na rede local da unidade (Intranet), optou-se pela utilização do padrão Wi-Fi (Wireless Fidelity) de redes sem fio, por permitir um alcance suficiente para a comunicação entre o servidor e o cliente na unidade. Adicionalmente foi escolhido o protocolo de segurança WEP (Wired Equivalent Privacy) para a autenticação de acesso. ${ }^{24}$

A escolha do equipamento móvel PDA ocorreu em função da disponibilidade como ferramenta de estudo do grupo técnico envolvido no projeto. Em função desta opção, todas as informações do 
presente aplicativo foram subdivididas em blocos compatíveis para formatação da tela do PDA. ${ }^{25}$

A elaboração do protótipo incluindo entrevistas, planejamento, programação de dados, diagramação e implantação na estação de trabalho (servidor) e no aplicativo móvel (cliente) ocorreu em quatro meses.

Na seqüência, foi realizado teste piloto com 12 pacientes, para treinamento da pesquisadora na operação do sistema e do equipamento. Os dados foram avaliados em relação à integralidade na transmissão do equipamento móvel para o servidor. Neste período, o NAS também foi coletado de forma manual, com anotação por uma enfermeira treinada pela pesquisadora. Os dados obtidos simultaneamente em papel e pelo sistema eletrônico mostraram concordância nas informações.

Assim, foi coletado o NAS, pela pesquisadora, diariamente durante a visita à beira do leito, pela manhã, durante 90 dias. Levantou-se informações referentes às necessidades dos pacientes, obtidas com a leitura dos registros realizados pela enfermagem nas últimas 24 horas. Deste modo, a coleta foi de aferição contemporânea sobre achados retrospectivos dos pacientes. O tempo de realização da leitura dos registros do paciente e inserção dos dados no sistema foi em média de 5 minutos por paciente.

Ao término da coleta de dados à beira do leito foi visualizada a distribuição do escore no sistema, para confirmação da transmissão e armazenamento dos dados. Adicionalmente, realizou-se a impressão diária de tabelas e gráficos gerados pelo sistema.

\section{APRESENTAÇÃO DOS RESULTADOS E DISCUSSÃO}

Passa-se a apresentar os dados referentes às etapas operacionais do aplicativo informatizado na sequência, com as interações dinâmicas no sistema e resultados relativos à carga de trabalho de enfermagem, segundo o NAS. ${ }^{8}$

\section{Características operacionais do aplicativo}

$\mathrm{O}$ acesso ao sistema foi feito pela senha de conexão na internet no provedor da Universidade. Os acessos são divididos em um login de usuário e um login de administrador, com senhas pessoais para acesso. A diferença principal entre os perfis de acesso encontra-se na página "Administrar Paciente", que permite o gerenciamento dos dados do paciente, sendo restrita ao administrador do sistema. A Figura 1 mostra a tela inicial com todas as possibilidades de acesso no sistema, composta de botões de acesso a dois grupos de páginas: I. Páginas de cadastro, coleta e armazenamento de dados e II. Páginas para consultas e análises dos dados armazenados.

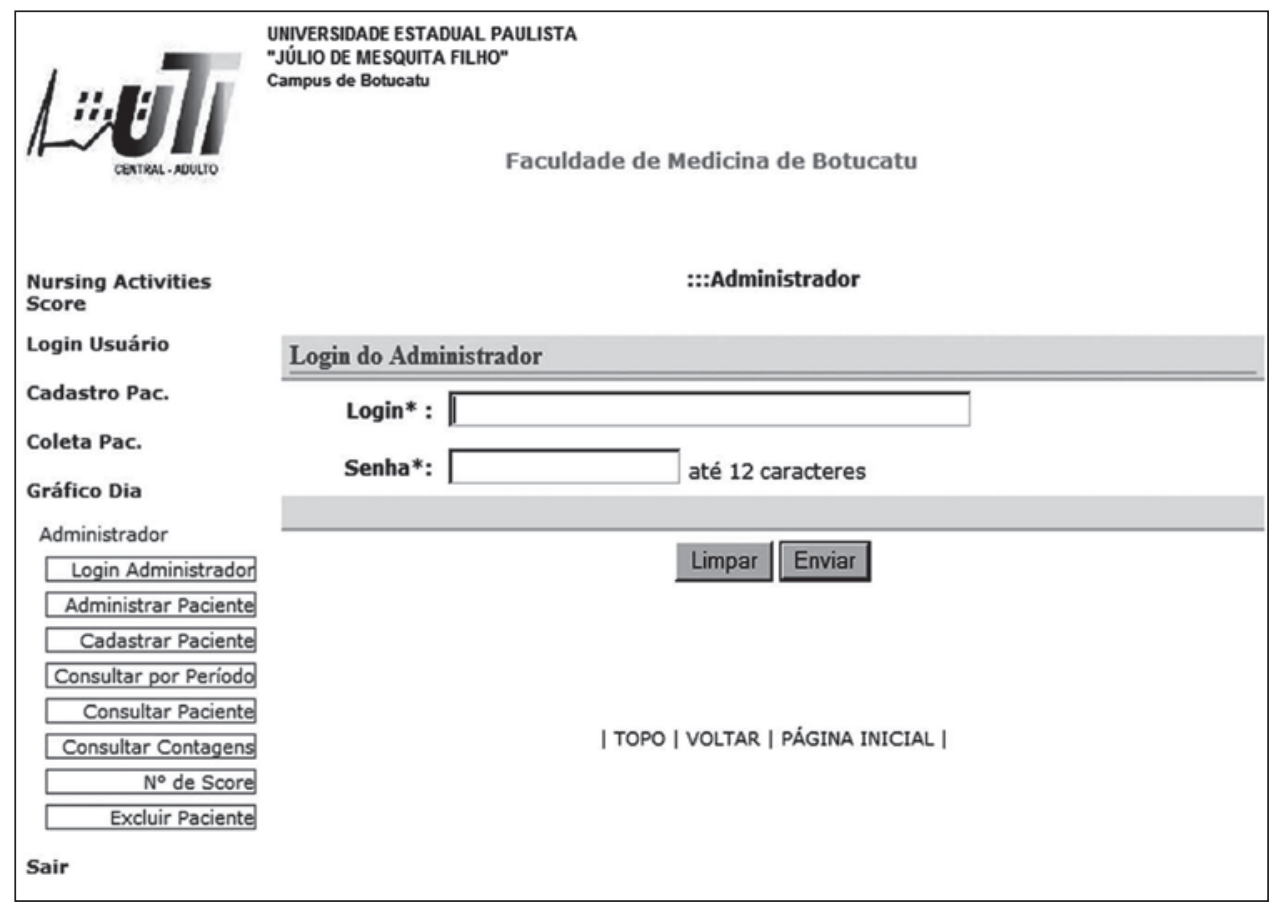

Figura 1 - Página de acesso para o administrador do aplicativo Nursing Activities Score na unidade de terapia intensiva 
Páginas de cadastro, coleta e armazenamento de dados

Administrador - acessa as opções "Paciente Alteração" (exclusivo do administrador), "Consulta Dia", "Consulta por Paciente", "Consulta Número de Score".

Login Usuário - insere novos pacientes e realiza coleta de dados.

Nursing Activities Score - apresenta breve descrição sobre o $\mathrm{NAS}^{8}$, definição do objetivo, utilização e proposta de implantação do aplicativo na UTI. Cita-se a referência sobre o escore e o contato com a pesquisadora deste trabalho. É recomendado que esta página seja a primeira a ser acessada pelo usuário, cumprindo a finalidade de apresentar em síntese o NAS.

Cadastro Paciente - acessado pelo usuário ou administrador, permite o registro do paciente.

Coleta Paciente - abre as páginas com o conteúdo do NAS, sendo um item por página, com opção de sim e não.

Seguindo-se sequencialmente a coleta dos dados do NAS, ao término aparece o valor final e automaticamente, será gerenciado tanto no registro do paciente como no registro do dia de coleta. O número do NAS é obtido pela soma de todos os itens. Exemplo: item $1+$ item $2+$ item 3 $+\ldots+$ item $23=$ valor do NAS. Cabe ressaltar que os itens com duas ou três escolhas são exclusivos, sendo que na escolha de um subitem, excluem-se os demais itens relacionados ${ }^{8-9} \mathrm{Na}$ construção do aplicativo informatizado esta exclusão foi viabilizada e ocorre de forma automática.

Administrar Paciente - mostra duas categorias de pacientes, habilitados (internados) e desabilitados (alta ou óbito).

Dados do Paciente - relaciona os escores diários em forma de tabela, dia a dia. Contém os dados de registro, número do leito, código do paciente no estudo (exemplo: paciente 1 , paciente 2), condição de habilitado (sim e não), especialidade, tipo de internação (clínica, cirúrgica eletiva, cirúrgica urgência) e situação de desfecho (alta ou óbito). Abaixo, aparece uma tabela com todos os dias de internação do paciente e seus respectivos NAS. Os dados revelam o histórico do paciente na unidade, estando ele internado ou não. Quando o paciente for desabilitado, suas informações estarão armazenadas no banco de dados dos pacientes desabilitados, com acesso na página "Administrar Paciente".

\section{Páginas para consultas e análises dos dados armazenados}

Na sequência aparece a página de consulta de dados individuais, pela seleção do registro do paciente (Figura 2). Este procedimento poderá ser usado como ferramenta para avaliação da carga de trabalho de cada paciente, facilitando o estudo de cada caso individualmente, podendo ser usada na análise do processo de trabalho.

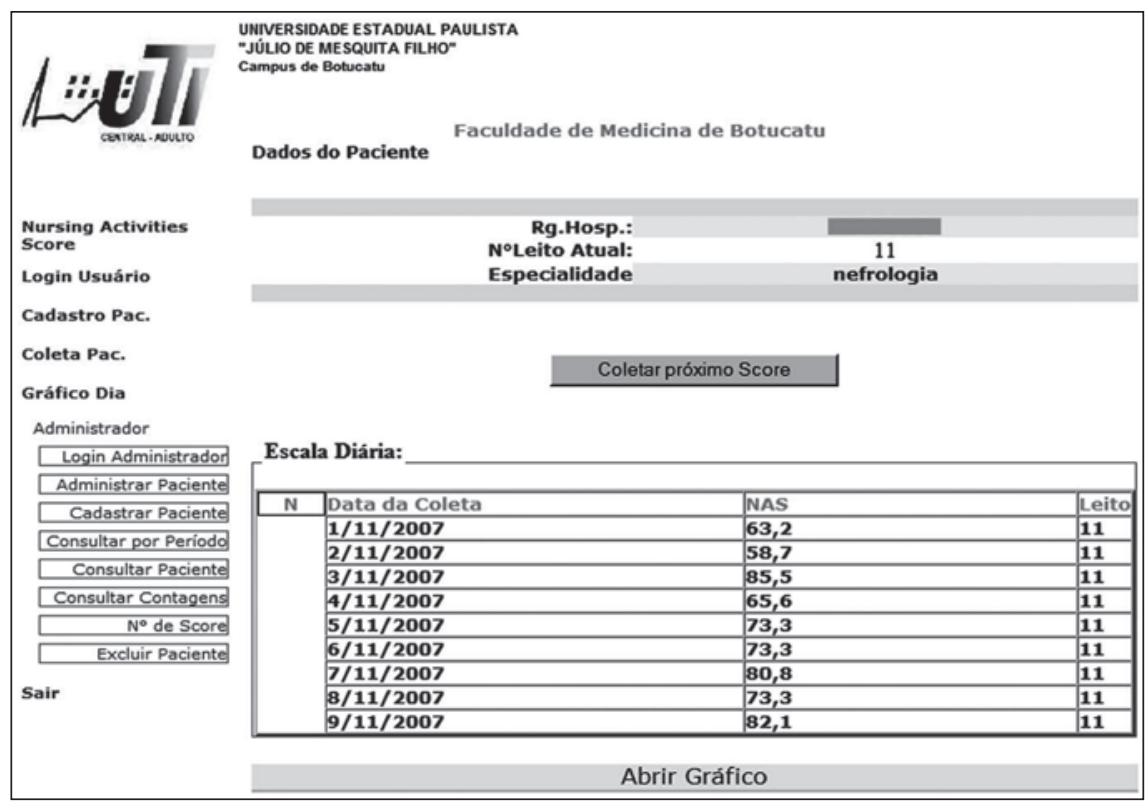

Figura 2 - Página demonstrativa com dados de um paciente internado na unidade de terapia intensiva, mostrando o valor do Nursing Activities Score em cada dia de internação 
Os dados anteriores transportados para um gráfico oferecem outra possibilidade de avaliação. No exemplo pode-se observar pouca variação da carga de trabalho de enfermagem no cuidado a um dado paciente. Entretanto, nos últimos dias de internação houve expressivo aumento da carga de trabalho de enfermagem que, no caso, coincidiu com o agravamento da condição clínica do paciente até sua saída da UTI. Assim, a aplicação simultânea de um índice de gravidade com o NAS, poderia auxiliar na hipótese de relação entre gra- vidade e carga de trabalho de enfermagem, como foi observado em alguns trabalhos. ${ }^{9-15}$

Consulta Pacientes do Dia - apresenta um resumo diário que possibilita avaliar a carga de trabalho do dia de toda a unidade. Apresenta a opção "Abrir Gráfico", que realiza a transferência dos dados para uma planilha no programa $\mathrm{Mi}$ crosoft Excel, formando um gráfico de colunas ou barras com todos os índices coletados no dia. Este formato permite uma visualização mais clara sobre a carga de trabalho na UTI (Figura 3).

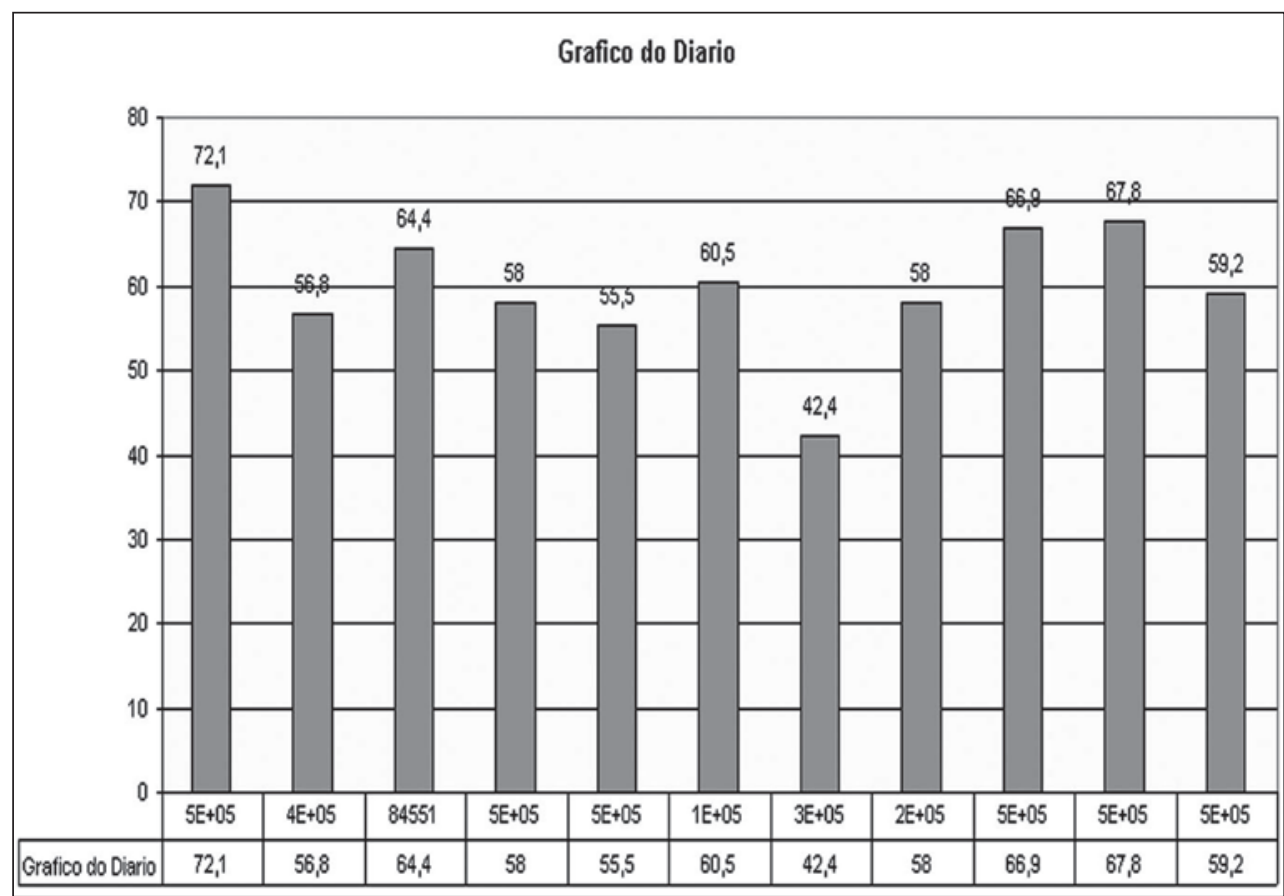

Figura 3 - Gráfico com os escores de todos os pacientes em determinada data no aplicativo Nursing Activities Score

Número de Escore - mostra o gerenciamento de todos os dados de todos os pacientes. Ao selecionar uma data, temos uma coorte de pacientes até o dia solicitado, podendo ser atual ou uma data pregressa. Como resultado é gerado tabela com os vinte e três itens e subitens do NAS ${ }^{8}$ e a respectiva contagem geral do índice. É possível saber quantas vezes cada item do NAS apareceu na população relacionada, incluindo pacientes habilitados e desabilitados. Seguem as opções em transferir dados para um gráfico de linha ou gráfico de colunas ou barras. Desta forma, pode-se conhecer as necessidades assistenciais de um grupo de pacientes, possibilitando ao enfermeiro intervenção no processo de gerenciamento da equipe. ${ }^{19}$
Após a avaliação em 12 pacientes, constatada a viabilidade e confiabilidade do sistema, passouse à coleta de dados em 90 dias consecutivos de com pacientes.

Dos resultados sumarizados com a caracterização da carga de trabalho de enfermagem segundo o $\mathrm{NAS}^{8}$ tem-se a média geral nos 123 pacientes com 61,9 , e desvio padrão de 15,5 ; mediana de 62,6, tendo variação de 27,8 a 123,1 nos casos de menor e maior NAS no estudo. Em estudos com diferentes delineamentos, mas que utilizaram o NAS como uma das aplicações metodológicas, encontramos a média geral do NAS variando entre 52,1 e 74,6.9-13,15 
O reconhecimento das características do cuidado que são apresentadas a partir do aplicativo, deve ser realizado pelo enfermeiro. Para isto, é necessário que o profissional seja treinado e capacitado para identificar as demandas assistenciais de acordo com sua realidade. ${ }^{26}$

Atualmente o aplicativo informatizado com o NAS é utilizado diariamente na unidade em que foi desenvolvido, por treze enfermeiros que receberam treinamento teórico e prático. Sua aplicação auxilia a equipe de enfermeiros a tomarem decisões relativas ao processo de cuidar, pois determina com critérios científicos, por exemplo, a divisão de trabalho da equipe de enfermagem. Permite além do dimensionamento de pessoal extrapolar essa conduta para outras decisões de ordem gerencial, assistencial e de pesquisa; monitoram assim a qualidade do serviço de enfermagem e pode ser um importante recurso no processo avaliativo em UTI.

Corroboram com essa afirmação as autoras que citam a necessidade de utilizar a informação sobre a clínica dos pacientes e sobre a assistência prestada que cresce na medida em que estes dados reflitam na qualidade da assistência, nas decisões do gerenciamento e nas pesquisas de enfermagem. ${ }^{27}$

A variação da carga de trabalho também foi observada nos diferentes dias da semana, agrupando-se o NAS de segunda-feira a domingo. Desta forma observou-se que a maior média do NAS foi encontrada às quintas-feiras $(62,8)$ e aos sábados $(64,0)$. As quintas-feiras correspondem aos dias em que são admitidos pacientes em pós-operatório de grandes cirurgias enquanto aos sábados aumentam-se as ocorrências de internações por grandes traumas, acidentes automobilísticos ou vítimas de violência. Assim estas evidências, segundo a dinâmica desta UTI, podem sugerir ao enfermeiro que mantenha um número de profissionais condizentes com esta realidade. Dentre tantas outras finalidades esses resultados permitem ao enfermeiro a condição para o desenvolvimento de ações que se direcione para melhoria na coordenação do processo de cuidar, de forma segura, alicerçado em ciência e não ao acaso.

\section{CONCLUSÃO}

Tendo em vista o objetivo proposto, os resultados obtidos no âmbito desse estudo, permitiram mostrar as etapas metodológicas para a construção do aplicativo informatizado utilizando-se o NAS. ${ }^{8}$ O estudo piloto com 12 pacientes foi capaz de assegurar a confiabilidade do sistema quanto à transmissão dos dados e garantiu o treinamento da pesquisadora. Também foi possível exemplificar a aplicabilidade com resultados sumarizados de 123 pacientes após 90 dias consecutivos de coleta. Adicionalmente, o aplicativo permitiu realizar a interface dos dados em composições gráficas, as quais facilitam a visualização.

Perante os dados sobre as características dos cuidados requeridos pelos pacientes evidencia-se que a utilização do aplicativo com o NAS poderá auxiliar nos processos de tomada de decisão no gerenciamento da unidade como o dimensionamento de pessoal e divisão da assistência. $\mathrm{Na}$ assistência, verifica-se que o instrumento facilitará no diagnóstico das necessidades clínicas dos pacientes, direcionando quais os itens que os caracterizam individualmente, como também a um grupo específico. Conhecendo essas características o enfermeiro tem a possibilidade de apropriar-se disso para o desenvolvimento de estratégias de educação permanente da equipe de enfermagem e correlacionar com indicadores gerenciais e assistenciais da unidade.

Pode-se fazer uma reflexão da construção do aplicativo informatizado com o conceito de tecnologia na dimensão de três camadas de significados assim abordadas, ${ }^{28}$ a do objeto físico, matéria que no estudo concretiza-se por meio de um sistema e utilização do PDA para o funcionamento; também a de uma forma de conhecimento, pois proporciona a utilização de um arcabouço teórico pelo NAS ${ }^{8}$ e outros conhecimentos necessários à prática em UTI e por fim faz parte de um conjunto complexo de atividades humanas o cuidado, gerência, educação e pesquisa. ${ }^{28}$

Por tratar-se de um hospital escola, o aplicativo foi utilizado pelo sistema de coleta e de armazenamento de dados, como fonte de informações e para o aprendizado da equipe de enfermagem da unidade. Pode também ser compartilhado com estudantes da graduação e pós-graduação, realinhando-se à missão da Universidade e da Enfermagem, que atua na assistência, ensino e pesquisa.

\section{REFERÊNCIAS}

1. Terzi RG, Gómez MI, Araújo S, Dragosavac D, Falcão AL, Machado HC. Índices prognósticos em medicina 
intensiva III: modelo UNICAMP. Rev Bras Terap Intensiva. 2002 Jan-Mar; 14(1):6-21.

2. Dias AT, Matta PO, Nunes WA. Índices de gravidade em Unidades de Terapia Intensiva adulto: avaliação clínica e trabalho da enfermagem. Rev Bras Terap Intensiva. 2006 Jul-Set; 18(3):276-80.

3. Knaus WA, Zimmerman JE, Wagner DP, Draper EA, Lawrence DE. APACHE - Acute physiology and chronic health evaluation: a physiologically based classification system. Crit Care Med. 1981 Aug; 9(8):591-7.

4. Le Gall JR, Loirat P, Alperovitch A, Glaser P, Granthil C, Mathieu D, et al. A simplified Acute Physiological Score for ICU patients. Crit Care Med. 1984 Nov; 12(11):975-7.

5. Teres D, Lemershow S, Avrunin JE, Pastides H. Validation of the mortality prediction model for ICU patients. Crit Care Med. 1987 Mar; 15(3):208-13.

6. Tranquitelli AM, Padilha KG. Sistemas de classificação de pacientes como instrumento de gestão em Unidades de Terapia Intensiva. Rev Esc Enferm USP. 2007 Jan-Mar; 41(1):141-6.

7. Ourcellón AA, Acunã L, Pilar M, Cancino U, Cecília A. Revisión de los sistemas de medición de intervenciones de enfermería utilizados en Unidades de Cuidado Intensivo. Rev Chil Med Intensiva. 2005; 20(4):221-6.

8. Miranda DR, Raoul N, Rijik A, Schaufeli W, Iapichino G. Nursing activities score. Crit Care Med. 2003 Fev; 31(2):374-82.

9. Queijo AF. Tradução para o português e validação de um instrumento para medida da carga de trabalho de enfermagem em unidade de terapia intensiva: Nursing activities score (NAS) [dissertação]. São Paulo (SP): Universidade de São Paulo. Escola de Enfermagem, 2002.

10. Conishi RMY, Gaidzinski RR. Nursing Activities Score - como instrumento de medida de carga de trabalho de enfermagem em UTI adulto. Rev Esc Enferm USP. 2007 Set; 41(3):346-54.

11. Dias MCCBH. Aplicação do Nursing activities score (NAS) como instrumento para medir a carga de trabalho de enfermagem em UTI cirúrgica cardiológica [dissertação]. São Paulo (SP): Universidade de São Paulo. Escola de Enfermagem; 2006.

12. Gonçalvez LA, Padilha KG. Fatores associados à carga de trabalho de enfermagem em unidade de terapia intensiva. Rev Esc Enferm USP. 2007 Dez; 41(4):645-52.

13. Silva MCM. Fatores relacionados com a alta, óbito e readmissão em unidade de terapia intensiva [tese]. São Paulo (SP): Universidade de São Paulo. Escola de Enfermagem; 2007.
14. Bochembuzio L, Gaidzinski RR Instrumento para classificação de recém-nascidos de acordo com o grau de dependência de cuidados de enfermagem. Acta Paul Enferm. 2005 Out-Dez; 18(4):382-9.

15. Ducci AJ, Padilha KG. Nursing activities score (NAS): estudo comparativo da aplicação retrospectiva e prospectiva em Unidade de Terapia Intensiva. Acta Paul Enferm. 2008 Out-Dez; 21(4):581-7.

16. Sousa CR, Gonçalves LA, Toffoleto MC, Leão K, Padilha KG. Preditores da demanda de trabalho de enfermagem para idosos internados em unidade de terapia intensiva. Rev Latino-am Enfermagem. 2008 Mar-Abr; 16(2):218-23.

17. Koerich MS, Backes DS, Scortegagna HM, Wall ML, Veronese AM, Zeferino MT, et al. Tecnologias de cuidado em saúde e enfermagem e suas perspectives filosóficas. Texto Contexto Enferm. 2006; 15(Esp):178-85.

18. Schramm FR, Escosteguy CC. Bioética e avaliação tecnológica em saúde. Cad Saúde Pública. 2000 OutDez; 16(4):951-61.

19. Santos MS, Martinez EYD. A introdução da informática na prática de enfermagem: possibilidade de mudanças na gestão do processo de trabalho. In: Anais do $8^{\circ}$. Simpósio Brasileiro de Comunicação em Enfermagem [Anais online]; 2002 Mai 2-3; São Paulo, SP, Brasil; 2002 [acesso 2008 Jan 16]. Disponível em: http://www.proceedings.scielo.br/scielo. php?script=sci_arttext\&pid=MSC00000000520020 00100016\&lng=en\&nrm=van

20. Marin HF, Marques EP. Nursing informatics: learning from the past to build a new future. Rev Bras Enferm 2005 Mar-Abr; 58(2):143-6.

21. Ministério da Saúde (BR). Portaria No 3432, de 12 de agosto de 1998. Estabelece critérios de classificação para as Unidades de Tratamento Intensivo - UTI. Diário Oficial da União, 13 Ago 1998. Seção I. p.108-10.

22. Alavi, M. An Assessment of the Prototyping Approach to Information System Development. CACM. 1984 Jun; 27(6):556-63.

23. L Burness, D Higgins, A Sago, P Thorpe. Wireless LANs - present and future. BT Technology J. 2003 Jul; 21(3):32-47.

24. Weissinger, A. Keyton, ASP: o guia essencial. Rio de Janeiro (RJ): Editora Campus; 2000.

25. Baumgart.DC Personal digital assistents in health care: experienced clinicians in the palm of your hand? Lancet. 2005 Oct; 366:1210-22.

26. Gonçalvez LA, Padilha KG. Nursing activities score (NAS): proposta para aplicação na prática em Unidades de Terapia Intensiva. Prat Hosp. 2005 Nov-Dez; 42:195-9.

27. Lundgrén-Laine H, Luominen T. Nursing intensity and patient classification at an adult intensive care 
unit (ICU). Intensive Crit Care Nurs. 2007 Apr; 23(2):97-103.

28. Dal Sasso GTM, Martins CR. Tecnologia: definições e reflexões para a prática em saúde e enfermagem. Texto Contexto Enferm. 2008 JanMar; 17(1):11-2.

Distrito de Rubião Junior, s/ $\mathrm{n}^{\circ}$

18618-970-Botucatu, SP, Brasil

E-mail: enfermeire.uti@fmb.unesp.br 